\title{
A New Drug Evaluation Method for the Treatment of Chronic Pancreatitis Using WBN/Kob Rats as a Model of Human Chronic Pancreatitis
}

\author{
Kazushi OHASHI*1 Ryoko ASO*1 Kazumasa NAKAMA*2 \\ and Toshio AKIMOTO*2
}

(Received on May 18)

*1 Clinical Pharmacology Center, Nippon Medical School

1-1-5 Sendagi, Bunkyo-ku, Tokyo 113-8602, Japan

*2 Division of Laboratory Animal Science, Nippon Medical School

\begin{abstract}
A new evaluation method for the treatment of chronic pancreatitis was devised using male WBN/Kob (WBN) rats as a human chronic pancreatitis model; in these rats, the lesion is spontaneously manifested at about 12 weeks of age. As a result of screening for drugs that inhibit the development of pancreatitic lesions in WBN rats, the inhibitory effects of estradiol (10 and $40 \mu \mathrm{g} / \mathrm{kg}$, i. p., twice a week), camostat mesylate ( $100 \mathrm{mg} /$ $\mathrm{kg}$, p. o., daily) and allopurinol ( $100 \mathrm{mg} / \mathrm{kg} \mathrm{p.} \mathrm{o.,} \mathrm{daily)} \mathrm{were} \mathrm{observed} \mathrm{among} \mathrm{estrogen,}$ testosterone lowering agent, trypsin inhibitors and depressors of active oxygen.

The efficacy of camostat mesylate and allopurinol which might be safely used for the treatment of progressive chronic pancreatitis were evaluated. Camostat mesylate was found to be effective, but not allopurinol. The expression of cells at the DNA synthesis stage as determined based on Brd Uridine (BrdU) uptake was examined. The uptake of BrdU notably occurred in acinar cells adjacent to the pancreatitic area. The regeneration of the pancreas as a result of the pancreatic trophic effect is the mechanism through which camostat mesylate is effective against lesions (mainly fibrosis) of chronic pancreatitis during the progression stage. One week after camostat mesylate administration, the weight of the pancreas increased rapidly, during which the uptake of BrdU in cells increased concurrently, indicating that regeneration of the pancreas occurred during this period.

The most effective parameter for evaluating the efficacy of a drug for pancreatitis is the percentage of pancreatitic area employed in our histopathological study. We concluded that the use of WBN rats is pharmacologically effective for drug evaluation.
\end{abstract}

Key words : male WBN/Kob rat, chronic pancreatitis, drug evaluation, trophic effect

\section{Introduction}

The mechanisms underlying the pathogenesis of chronic pancreatitis, for which an effective treatment method has not been established, remain largely unclarified. In order to clarify the mechanisms and develop a drug for chronic pancreatitis, the development of chronic pan-

*1 日本医科大学臨床薬理センター $\mathbf{T} 113-8602$ 東京都文京区千駄木 1-1-5, ${ }^{* 2}$ 日本医科大学実験動物部門 
creatitis model animals has been desired. We obtained histopathological findings similar to chronic pancreatitis before glycosuria was observed in male WBN/Kob (WBN) rats which we had studied as a spontaneously occurring diabetic model ${ }^{1)}$ and reported the characteristics of WBN rats $^{2)}$. Pancreatic histopathological findings of our male WBN rats satisfied the criteria for the classification of pancreatitis $^{3)}$ proposed at the Marseille conference and the clinical diagnostic criteria of chronic pancreatitis ${ }^{4}$ proposed by the Japanese Society of Gastroenterology (1983). Accordingly, we considered that the WBN rat is a spontaneously occurring human chronic pancreatitis model.

The aim of this study was to investigate whether or not WBN rats could be used as a model of human chronic pancreatitis for evaluating the efficacy of a drug for chronic pancreatitis. To examine these requirements, drugs that were considered to be effective in treating pancreatitic lesions in WBN rats were screened. Those found to be effective after the screening test were further tested for their effectiveness against pancreatitis at the initial and progression stages. Furthermore, mechanisms by which the tested drugs act on the disease were also studied.

\section{Experimental materials}

A total of 192 male WBN rats ( 8 and 15 weeks old) were employed as specimens. Rats of both age groups were purchased one week prior to the experiment and reared under the following conditions: room temperature $23 \pm$ $3^{\circ} \mathrm{C}$, humidity $55 \pm 10 \%$ and illumination $14 \mathrm{~h}$.

Drugs tested : Camostat mesylate (Foipan ${ }^{\circledR}$, Ono Pharmaceutical Co., Ltd.) and allopurinol (Zyrolic ${ }^{\circledR}$, Sumitomo Pharmaceuticals, Co., Ltd.) were mixed with the feed which was administered to the rats; the amount of feed intake per day for rats at 8 weeks old (weighing approximately $200 \mathrm{~g}$ ) was determined to be 20 g. Each drug was administered peroraly at a dose of $100 \mathrm{mg} / \mathrm{kg}$ body weight/day. Estradiol (Ostradiol ${ }^{\circledR}$, Merck), a luteinizing-hormonereleasing hormone (LH-RH) sustained-release tablet (Leuplin ${ }^{\circledR}$, Takeda Chemical Industries Ltd.), superoxide dismutase (SOD, Sigma Chemical) and catalase (Sigma Chemical) were subcutaneously injected.

\section{Methods}

The protocol of this study was approved by the Animal Experimental Ethical Review Committee of Nippon Medical School (April 7, 1993) in accordance with the "Guideline for Animal Experimentation in Nippon Medical School".

\section{Histopathological study, pancreatic func- tional study, and examination of activity of pancreatic enzyme and hormone in blood.}

To perform the histopathological study, peritoneotomy was performed on the WBN rats under ether anesthesia, and after blood was collected from the aorta abdominalis, the pancreas was excised and weighed, and then fixed with Bouin's solution. The pancreatic corpus was extended in the form of a leaf. Then, the pancreas was cut into three blocks which were then embedded in paraffin according to a conventional method. Each pancreatic block was cut into $4-\mu \mathrm{m}$ thick sections. Among the thinly sliced specimens of each pancreatic block, two specimens with the greatest area were stained with hematoxylin eosin (HE) and Gomori's aldehyde-fuchsin (AF) and studied microscopically.

To study the pancreatic function, the exocrine pancreatic function diagnostant (PFD) test was performed ${ }^{5)}$. After fasting, sastargen 
( $0.38 \mathrm{mg} / \mathrm{kg}$ body weight) and BT-PABA (10 $\mathrm{mg} / \mathrm{kg}$ body weight) were administered through a stomach tube. The excretion ratio of BTPABA in $6 \mathrm{~h}$ cumulative urine was calculated.

To study activities of enzymes in the pancreas, serum amylase, lipase, elastase and trypsin activities were measured. To determine hormone activity in blood, levels of immunoreactive insulin (IRI), testosterone and corticosterone were measured. Enzyme activities and hormone levels in blood were measured at Mitsubishi Chemical BCL Corp. using a commercially available kit. Statistical analyses of differences in PFD test result between before and after the treatment. While those in pancreatic enzyme activity and IRI concentration between the treated and non-treated groups were performed using Student's t-test.

\section{Pharmacological evaluation}

1) Screening of drugs which inhibit development of pancreatitis

Drugs were administered to the 8- to 15 -weekold rats for 8 weeks, during which the initial stage of pancreatitis was observed. Drugs which were considered to inhibit the development of pancreatitis were screened. The 8 weeks old male WBN rats were categorized into 5 drug-administration groups [ (A), (B), (C), (D) and (E) (4 rats for each group)] and the non-treated group (F) (4 rats). The exocrine PFD study, administration of drugs and the histopathological study were carried out according to the following schedule:(1) The initial PFD test was performed on rats at 8 weeks old before the administration of drugs.

(2) Drugs were administered for 8 weeks. ( 3 ) Two days after the completion of drug administration, the PFD test was performed following the method described in (1). (4) Two days after (3), blood was collected from the animals, which were then sacrificed and autopsied.

In the histopathological study, the severity of chronic pancreatitis was evaluated based on the extent of pancreatitic expansion. From each of the 3 pancreatic blocks of individual rat, a representative section was obtained and observed under a low magnification (10-15 x). For evaluation, the ratio of the area with pancreatitis to that of the entire tissue specimen was categorized into 4 groups $0-25 \%, 26-50 \%$, $51-75 \%$ and $76-100 \%$. In determining the severity of the pancreatitic lesion in each rat, the median value of the 3 sections which represent the entire area of the pancreas, was used as the percentage of pancreatitic area of the rat.

The severity of histopathological pancreatitis was categorized as follows : $76-100 \%$ indicating very severe pancreatitis, $51-75 \%$ severe pancreatitis, $26-50 \%$ moderate pancreatitis and $0-25 \%$ borderline pancreatitis. Based on these categories, the efficacy of drugs was evaluated. Given the observation that the percentage of pancreatitic area in the nontreated group indicated a severe or very severe pancreatitis at $50 \%$ or more, when the percentage of pancreatitic area was $25 \%$ or less following the administration of a drug, the drug was determined to be very effective, and when the percentage of pancreatitic area was $26-50 \%$, the drug was determined to be effective.

2 ) Study of the drug administration timing related to treatment (treatments during the initial and progression stages)

Clinically safe drugs were selected among the drugs determined to be effective in inhibiting the formation of pancreatitic lesions in the WBN rats after the screening tests. We studied, whether or not these drugs were effective in inhibiting pancreatitis during the 8 weeks of drug administration in male WBN rats from 8 to 15 weeks old which were at the initial stage 
of pancreatitis and those from 18 to 25 weeks old which were at the progression stage of pancreatitis. The efficacy of the selected drugs was determined using the same method as that used in the screening test. For the initial and progression stages, the nontreated groups of 6 and 7 rats were used as the control groups, respectively. The percentage of the cases exhibiting a lower than $50 \%$ pancreatitic area with respect to the total number of administered cases was determined as a measure of the effective percentage. Evaluation of the drugs was performed using a $\chi^{2}$ test of the effective percentages of drugs in the treated and nontreated groups.

\section{3 ) Study on pancreatic regeneration}

(1) Expression and distribution of cells exhibiting BrdU uptake: Brd Uridine (BrdU) (15 $\mathrm{mg} / \mathrm{kg}$ body weight) was administered to the abdominal cavity $20 \mathrm{~min}$ prior to the sacrifice. Cells at the DNA synthesis stage obtained from the excised pancreas were marked according to the method described by Sasaki et $\mathrm{al}^{6}$. Expression and distribution of cells exhibiting BrdU uptake in the pancreas of WBN rats at 16 weeks old in the nontreated group and those at 25 weeks old, that is, 8 weeks after administration of camostat mesylate $(100 \mathrm{mg} / \mathrm{kg}$ body weight/day), were studied.

(2) Time course of pancreatic regeneration after administration of drugs: To study the efficacy of a drug against pancreatitis which has a pancreatic trophic effect with time, the peroral trypsin inhibitor, camostat mesylate (100 mg/kg body weight/day) was administered to the WBN rats at 25 weeks Determination of the weight of the pancreas, and BrdU uptake and histopathological studies were performed on 2 rats at $3,7,10,14,21,28$, and 42 days after the administration and on rats of the nontreated group.

\section{Results}

\section{Pharmacological evaluation}

1) Screening of drugs which inhibit development of pancreatitis

Pancreatitic lesions in male WBN rats can be observed macroscopically as localized brown lesions in the pancreas when the rats were 3 months old, then the lesions coalesce to cover the entire pancreas. By light microscopy, acinar interstitial edema, hemorrhage and infiltration of inflammatory cells and fibroblasts were observed in the brown lesions of the rats at 3 months old (Fig. 1-a). With aging, fibrosis developed with necrosis and the loss of the pancreatic parenchyma (Fig. 1-b). After 16 weeks old, distention of the pancreatic duct with ductal epithelial metaplasia and periductal fibrosis, proliferation of small pancreatic ducts in the parenchymal fibrotic area and fragmented images of islets were observed (Fig. 1-c), which gradually deteriorated further. For visual comparison, the normal pancreas of a female WBN rat is shown in Fig. 1-d.

(1) Non-treated (control) group : The pancreatic specimens (Fig. 2-f) from all 4 rats at 16 weeks old in the non-treated group exhibited a pancreatitic area larger than $51 \%$ (Table 1). Therefore, when the percentage of pancreatitis was lower than $50 \%$ following the administration of a drug in the screening test, we determined the drug to be effective in treating pancreatitis.

(2) Estradiol-treated group : Estradiol was administered intraperitoneally twice a week. Depending on the administered amount, the rats were categorized into a high-estradiol group (40 $\mu \mathrm{g} / \mathrm{kg}$ body weight) and a low-estradiol group (10 $\mu \mathrm{g} / \mathrm{kg}$ body weight). In the high-estradiol group, the PFD level $(79.5 \pm 7.9 \%)$ was significantly higher than that before the administra- 

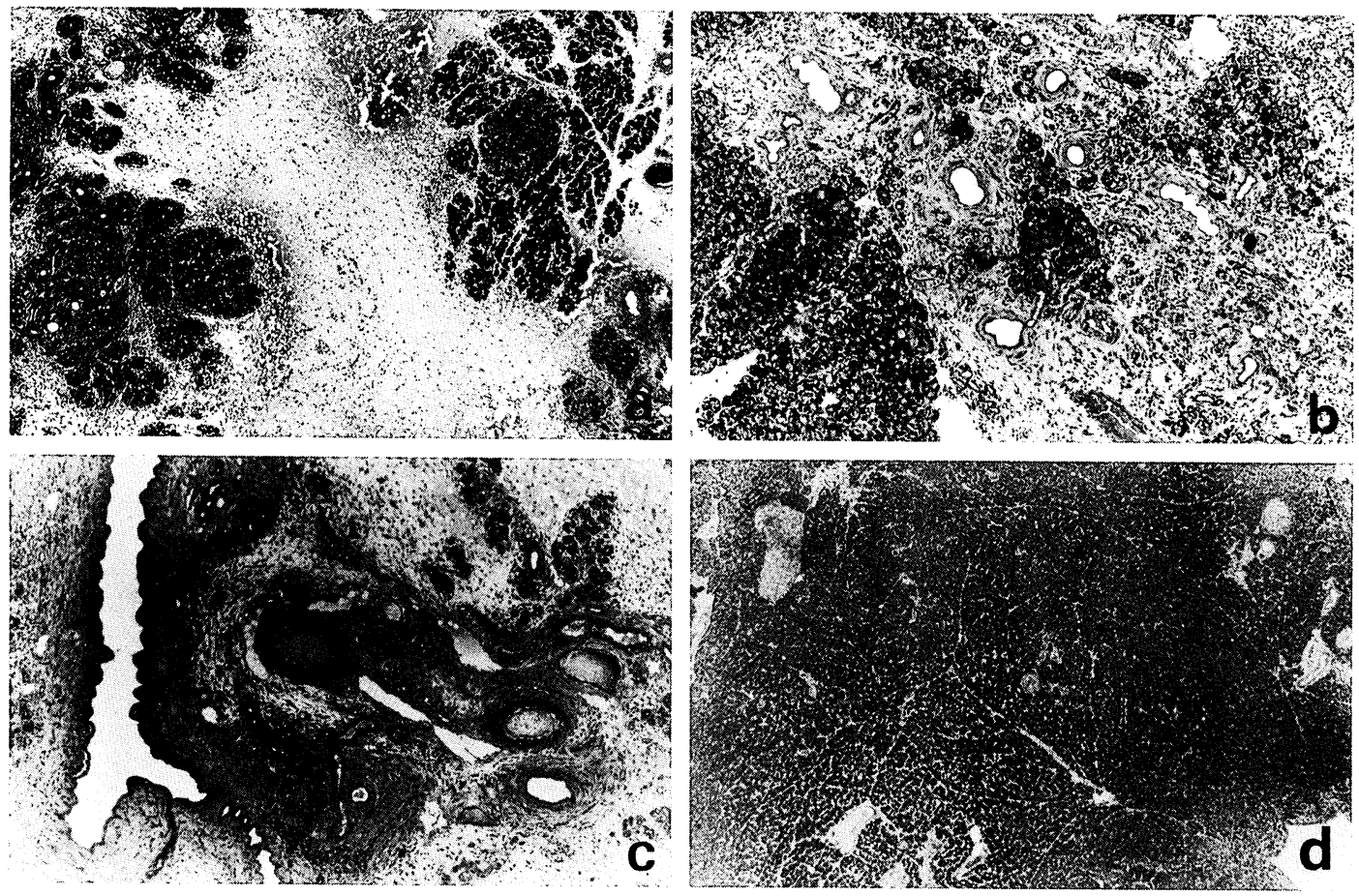

Fig. 1 Histopathological observations of the pancreas in male WBN/Kob rats showing: (a) infiltration of the inflammatory cells with interstitial edema and hemorrhage at 3 months old, $\mathrm{HE}(\times 40),(\mathrm{b})$ marked inter-and intralobular fibrosis with ductular proliferation and isolation of islets, $\mathrm{HE}(X$ 40 ), (c) dilatation of pancreatic duct with periductal fibrosis and smaller ductular proliferation at 9 months, HE $(\times 40)$, (d) a comparison with the normal pancreas of female WBN/Kob rats at 3 months old, $\mathrm{HE}(\times 40)$.

tion $(61.8 \pm 2.8 \%$ ) (Table 2$)$. Although no significant differences in the levels of serum pancreatic enzyme series and IRI were observed, the level of serum corticosterone after the administration was significantly different between the control and treated groups (Table 2). The testosterone level tended to decrease after the administration with no statistical significance (Table 2). Histopathologically, localized degeneration such as cellular infiltration was observed (Fig. 2-a). In the estradioltreated group, 2 rats exhibited a $26-50 \%$ pancreatitic area, and another 2 rats a $0-25 \%$ pancreatitic area, indicating that the development of pancreatitic lesion was generally inhibited (Table 1). No changes in the weight of the pancreas were observed between the treated $(-741.0 \pm 81.9 \mathrm{mg})$ and non-treated (772.3 \pm 71.1 $\mathrm{mg}$ ) groups (Table 2). Administration of estradiol at low amounts $(10 \mu \mathrm{g} / \mathrm{kg}$ body weight) also resulted in improvement in the exocrine PFD level (Table 2) and histopathological improvements (Table 1 ). Corticosteron level did not increase as observed in the high erstradiol group (Table 2).

(3) LH-RH-treated group : A $3 \mathrm{mg} / \mathrm{kg}$ body weight LH-RH sustained-release tablet was inserted subcutaneously once in 4 weeks. Two rats exhibited a $51-75 \%$ pancreatitic area and another 2 rats a $76-100 \%$ pancreatitic area (Table 1). LH-RH did not inhibit the development of pancreatitic lesions (Fig. 2-b). No sig- 

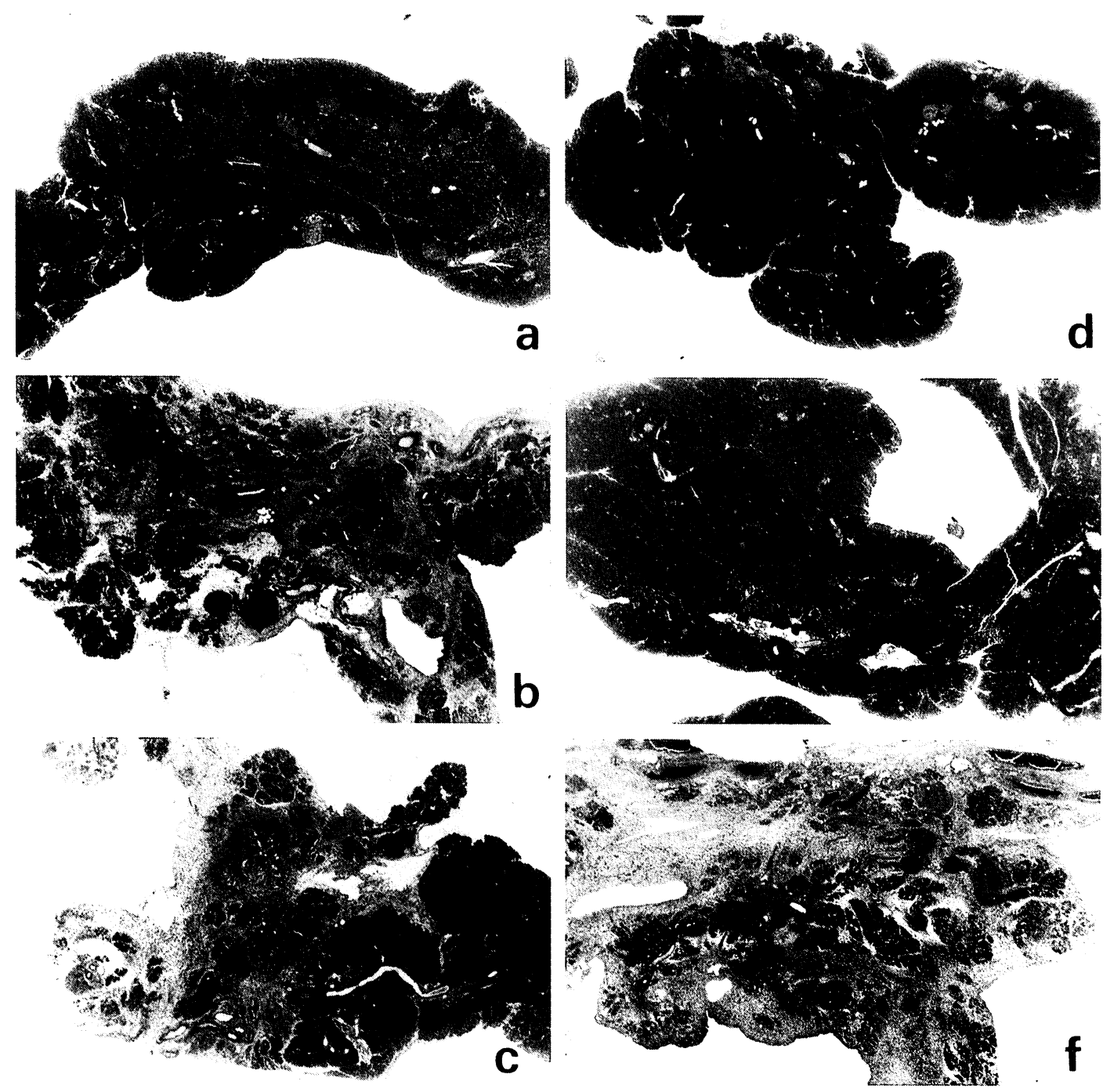

Fig. 2 Histopathological observations of the pancreas in male WBN/Kob rats at 16 weeks of age following treatment (from 8 to 15 weeks) of : (a) $40 \mu \mathrm{g} /$ body estradiol (sc, twice a week) ( $\times 10),($ b) $3 \mathrm{mg} / \mathrm{kg} \mathrm{LH} \cdot \mathrm{RH}$ (sc, every 4 weeks) $(\times 10),(\mathrm{c}) 50 \mathrm{mg} / \mathrm{kg}$ superoxide dismutase plus $10 \mathrm{mg} / \mathrm{kg}$ catalase (sc, twice a week) ( $\times 10)$, (d) $100 \mathrm{mg} / \mathrm{kg}$ allopurinol (po, daily) $(\times 10)$, and (e) $100 \mathrm{mg} / \mathrm{kg}$ camostat mesylate (po, daily) $(\times 10)$, compared to $(\mathrm{f})$ non-treated pancreas $(\times 10)$ as the control group. The percentages of pancreatitic area observed were (a) $0-25 \%$, (b) $76-100 \%$, (c) $51-75 \%$, (d) $0-25 \%$, (e) $0-25 \%$ and (f) $76-100 \%$, respectively.

nificant difference in the level of PFD was observed between the control and treated groups. The testosterone level in blood was decreased to $1.93 \pm 1.1 \mathrm{ng} / \mathrm{ml}$ which was onehalf that in blood of the nontreated group $(3.8 \pm$

\section{$2.6 \mathrm{ng} / \mathrm{ml}$ ) (Table 2).}

(4) SOD-catalase combined-treated group : superoxide dismutase (SOD) $(50 \mathrm{mg} / \mathrm{kg}$ body weight) and catalase (10 $\mathrm{mg} / \mathrm{kg}$ body weight) were separately dissolved in $0.5 \mathrm{ml}$ of physio- 
Table 1 Screening of Drugs Effecting the Percentage of Pancreatitic Area in the Microscopic Field

\begin{tabular}{|c|c|c|c|c|c|c|c|}
\hline $76 \sim 100$ & 00 & & & 00 & 00 & & \\
\hline $51 \sim 75$ & 00 & & & 0 & 00 & & \\
\hline $26 \sim 50$ & & 0 & 00 & & & & 0 \\
\hline $0 \sim 25$ & & 0 & 0 & & & & \\
\hline $\begin{array}{l}\text { Pancreatitic } \\
\text { area } \\
\text { (\%) }\end{array}$ & $\begin{array}{l}\text { non- } \\
\text { treated }\end{array}$ & $\begin{array}{l}\text { Estradiol } \\
(40 \mu \mathrm{g} \\
/ \text { body, } \\
\text { twice a } \\
\text { week),i.p. }\end{array}$ & $\begin{array}{l}\text { Estradiol } \\
(10 \mu \mathrm{g} \\
/ \text { body, } \\
\text { twice a } \\
\text { week),i.p. }\end{array}$ & $\begin{array}{l}\mathrm{LH}-\mathrm{RH} \\
\text { (3mg/kg, } \\
\text { once a } \\
\text { month), } \\
\text { s.c. }\end{array}$ & $\begin{array}{l}\mathrm{SOD} \\
(50 \mathrm{mg} / \mathrm{kg}, \\
\text { twice a } \\
\text { week }) \\
+ \text { Catalase } \\
(10 \mathrm{mg} / \mathrm{kg} . \\
\text { twice a } \\
\text { week),s.c. }\end{array}$ & \begin{tabular}{|l|} 
Allopurinol \\
$(100 \mathrm{mg} / \mathrm{kg}$ \\
$/$ day),p.o.
\end{tabular} & $\begin{array}{l}\text { Camostat } \\
(100 \mathrm{mg} / \mathrm{kg} \\
\text { /day),p.o. }\end{array}$ \\
\hline
\end{tabular}

logical saline, and administered twice a week subcutaneously. The level of PFD was not significantly changed (Table 2) and the percentage of pancreatitic area was larger than $50 \%$ (Fig. $2-c$, Table 1), indicating that no functional or histopathological improvement occurred.

(5) Allopurinol-treated group : Allopurinol (100 mg/kg body weight/day) was perorally administered daily. The PFD level was significantly increased after the administration compared to the before (Table 2). Other than the significant increase in the serum amylase level and the significant decrease in the IRI level, no statistically significant differences in the pancreatic enzyme series or hormone levels were observed between the treated and control groups (Table 2). Histopathologically, a localized inflammatory area was observed in the treated group; however, the pancreatic parenchyma remained intact (Fig. 2-d). Two rats exhibited a lower than $25 \%$ pancreatitic area and another 2 rats a $25-50 \%$ pancreatitic area (Table 1). From these results, we determined that allopurinol is effective in inhibiting the development of pancreatitis. The weight of the pancreas after treatment $(1079.9 \pm 68.2 \mathrm{mg})$ increased compared to that in the non-treated group (772.3 $\pm 71.1 \mathrm{mg}$ ) (Table 2).

(6) Camostat-mesylate-treated group : Camostat mesylate (100 mg/kg body weight/ day) was administered. A significant increase in the exocrine PFD level (from 49.8 to 99.0 ) was observed between before and after the administration to the rats from 8 to 15 weeks old (Table 2), indicating that the pancreatic exocrine function was improved. However, no significant differences in the serum pancreatic enzyme series levels were observed between the treatment and control groups. IRI levels were significantly decreased, while other hormone levels did not significantly change, compared to those of control groups (Table 2). Histopath- 


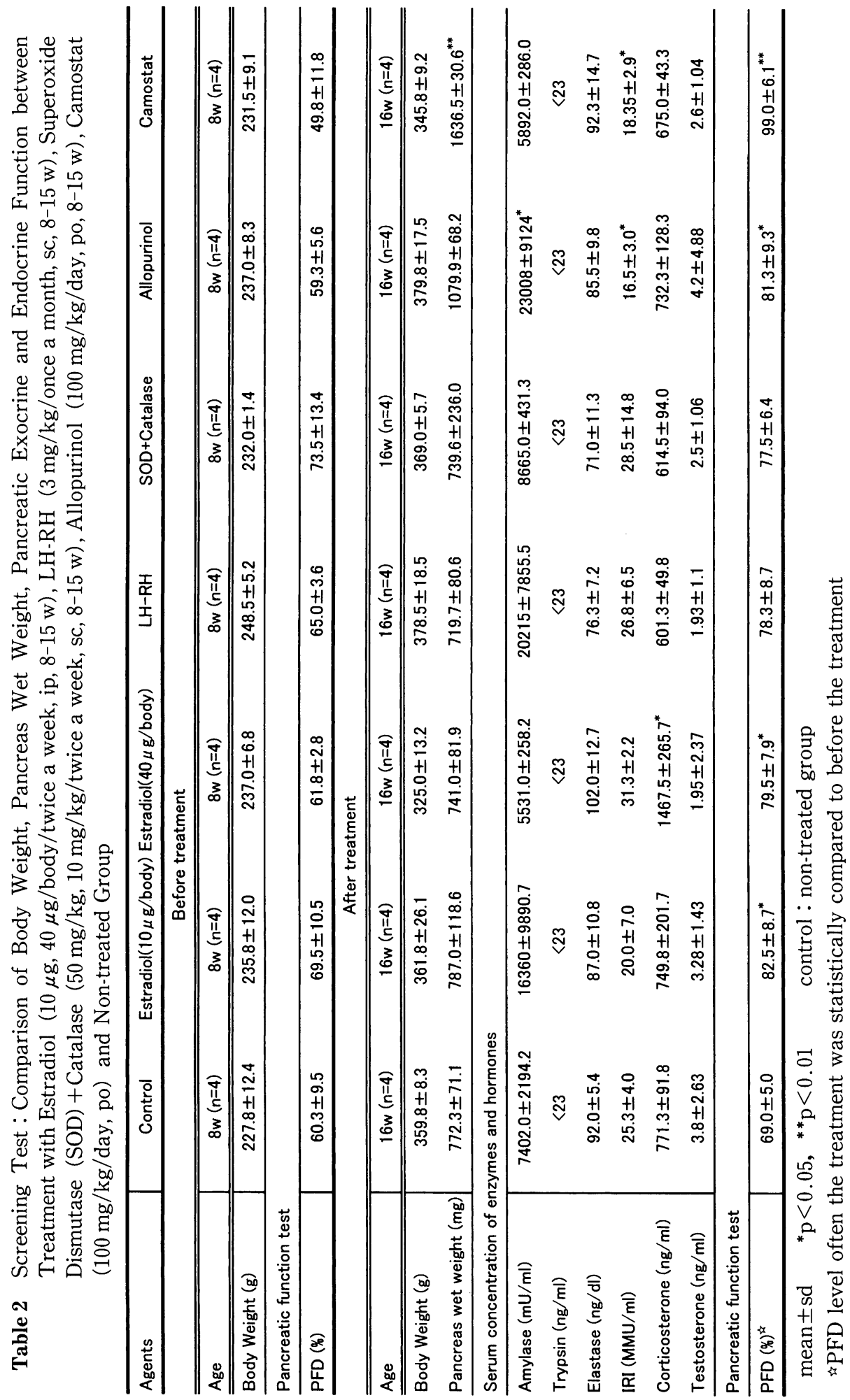




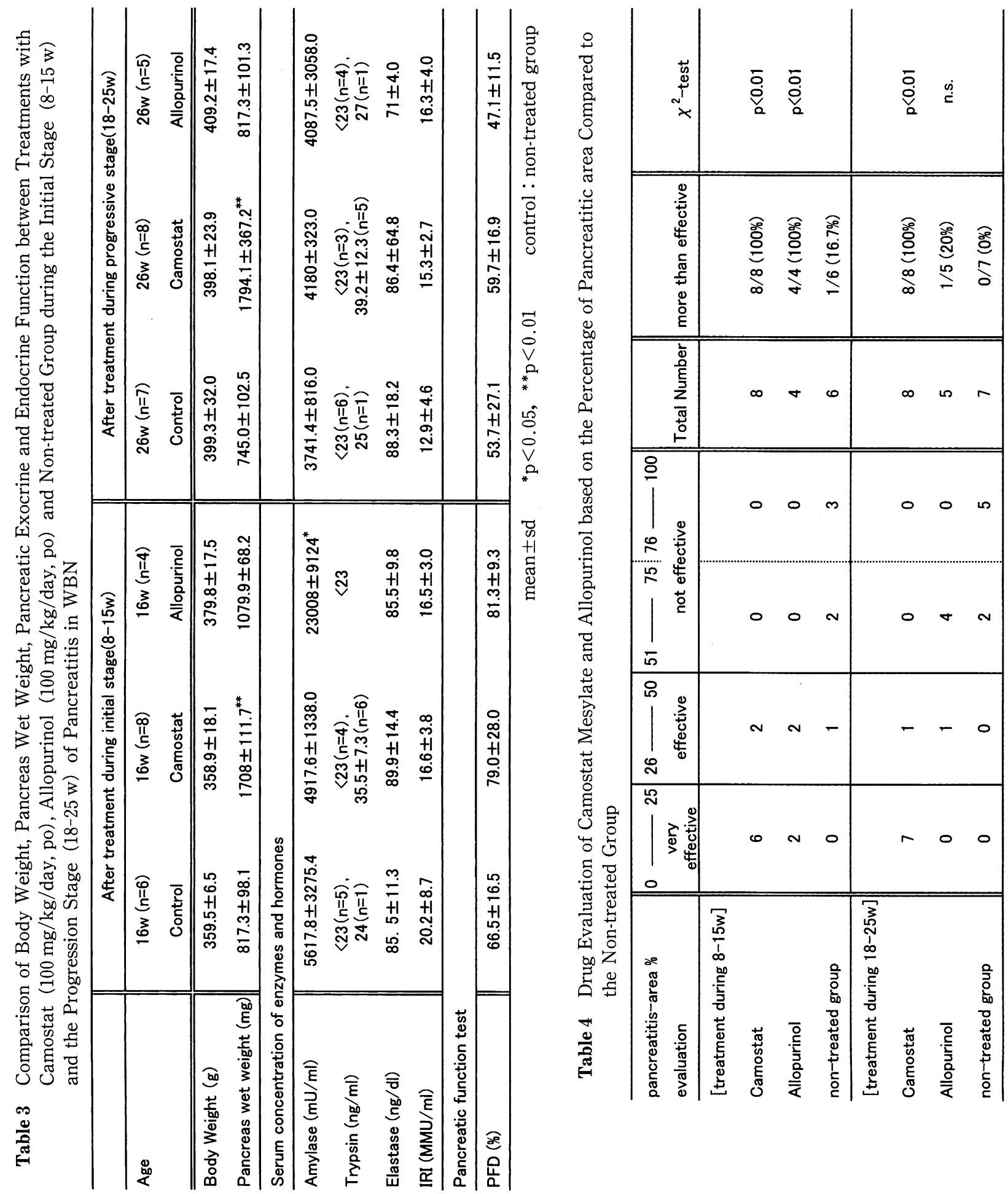


ologically, 3 of the 4 rats exhibited a lower than $25 \%$ pancreatitic area (Table 1), indicating that the development of pancreatitic lesions is strongly inhibited by camostat mesylate (Table 1). Histologically, hypertrophy of acinar cells was observed as shown in Fig. 2-e. The weight of the pancreas $(1636.5 \pm 30.69 \mathrm{mg})$ increased two-fold compared to that in the nontreated group (772.3 $\pm 71.1 \mathrm{mg}$ ) (Table 2). Accordingly, peroral administration of camostat mesylate inhibited pancreatitis and resulted in improvement of the pancreatic exocrine function.

From the above-mentioned results, it can be understood that camostat mesylate, estradiol, and allopurinol inhibited the development of pancreatitis in the WBN rats.

\section{2 ) Study on drugs for treatment (treatments} during the initial and progression stages)

Among the drugs determined to be effective by the screening test, estradiol was clinically unsuitable because of safety problems; thus only camostat mesylate $(100 \mathrm{mg} / \mathrm{kg}$ body weight/day) and allopurinol $(100 \mathrm{mg} / \mathrm{kg}$ body weight/day) were further tested for their clinical efficacy.

Results of treatment during the initial stage are shown in Table 3 and Table 4. Data from the cases of the screening test were added to those of the treatment study during the initial stage, because both examinations were carried out using the same dosing schedule. Among 6 rats in the nontreated group, 3 rats exhibited very severe pancreatitis, two had severe pancreatitis and one had moderate pancreatitis. Among 8 rats in the camostat mesylate-treated group, 6 rats exhibited a $0-25 \%$ pancreatitic area, indicating that the drug is very effective, and 2 rats a $26-50 \%$ pancreatitic area, indicating that the drug is effective (Table 4 ). In the allopurinol-treated group, the drug was very effective in 2 rats and was effective in another
2 rats (Table 4 ). Both drugs showed a $100 \%$ of the efficacy rate, respectively.

In the treatment during the progression stage, in the non-treated WBN rats at 26 weeks old, loss of the parenchyma with periductal fibrosis, irregular small pancreatic duct proliferation and periductal fibrosis developed from 16 weeks old, representing a condition typical of chronic pancreatitis (Fig. $3-\mathrm{c}$ ). Among 7 rats in the non-treated group, 5 rats exhibited a pancreatitic area larger than $76 \%$ and $51-75 \%$ in 2 rats (Table 4 ), these rats showed very severe and severe lesions, respectively. In addition, in the non-treated group, the average weight of the pancreas decreased with aging (Table 3), indicating a gradual pancreatic atrophy.

Meanwhile, among 8 rats in the camostatmesylate-treated group during the progression stage, 7 rats exhibited a lower than $25 \%$ pancreatitic area, indicating that the drug was very effective as the histological photo shows in Fig. 3-a. And 1 rat had a $26-50 \%$ pancreatitic area, indicating that the drug was effective. These results indicate that camostat mesylate was $100 \%$ effective in all rats (Table 4). The rat pancreatic tissue to which camostat mesylate was administered clearly exhibited hypertrophy of acinar cells (Fig. 3-d) compared to the non-treated group (Fig. 3-f). The weight of the pancreas in this group increased even with the administration of the drug during the progression stage and became approximately 2.7-fold higher than that in the non-treated group. No significant differences in the serum enzyme, PFD or IRI levels were observed between the initial and progression stages in the non-treated group (Table 3).

Among 5 rats in the allopurinol-treated group during the progression stage, one rat exhibited a $26-50 \%$ pancreatitic area indicating that the drug is effective; however 4 rats exhibited a 

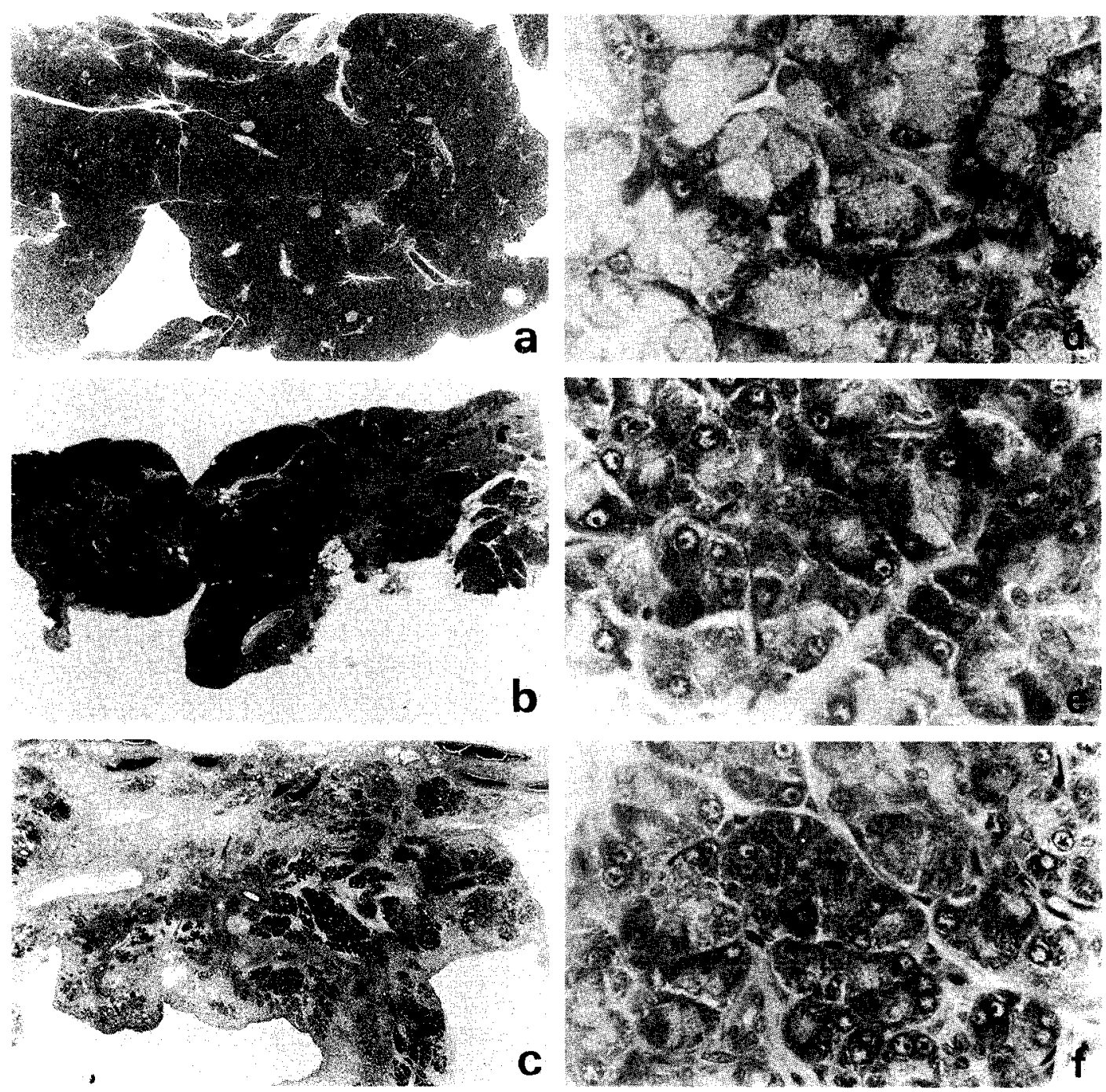

Fig. 3 Histopathological observations of the pancreas in male WBN/Kob rats at 26 weeks old following the therapeutic administration from 16 to 25 weeks of (a) $100 \mathrm{mg} / \mathrm{kg}$ camostat mesylate (po, daily) $(\times 10)$, (b) $100 \mathrm{mg} / \mathrm{kg}$ allopurinol (po, daily) $(\times 10)$ and $(\mathrm{c})$ non-treated pancreas as the control group $(\times 10)$. The percentages of pancreatitic area were : (a) $0-25 \%$, (b) $51-75 \%$ and (c) $76-100 \%$, showing camostat mesylate was very effective, while allopurinol was not effective. Camostat was effective, while allopurinol was not effective, comparing to (c) non-treated pancreas $(\times 10)$. (d), (e), (f) are large atitude microscopic photos of acinar cells with same scale $(\times 400)$ of camostat-, allopurinol-and non-treated WBNs, respectively. Growing of acinar cells is observed in (d), while not in (e), comparing to (c).

$51-75 \%$ pancreatitic area indicating the inefficacy of the drug. The efficacy percentage of allopurinol in this group was $20 \%$ which was not significantly different from that in the non-treated group (Table 4). Histopathological$1 y$, the pancreatic acinus had a normal shape in part ; however, pancreatitic lesions were observed in many places (Fig. 3-b). Hypertrophy of 

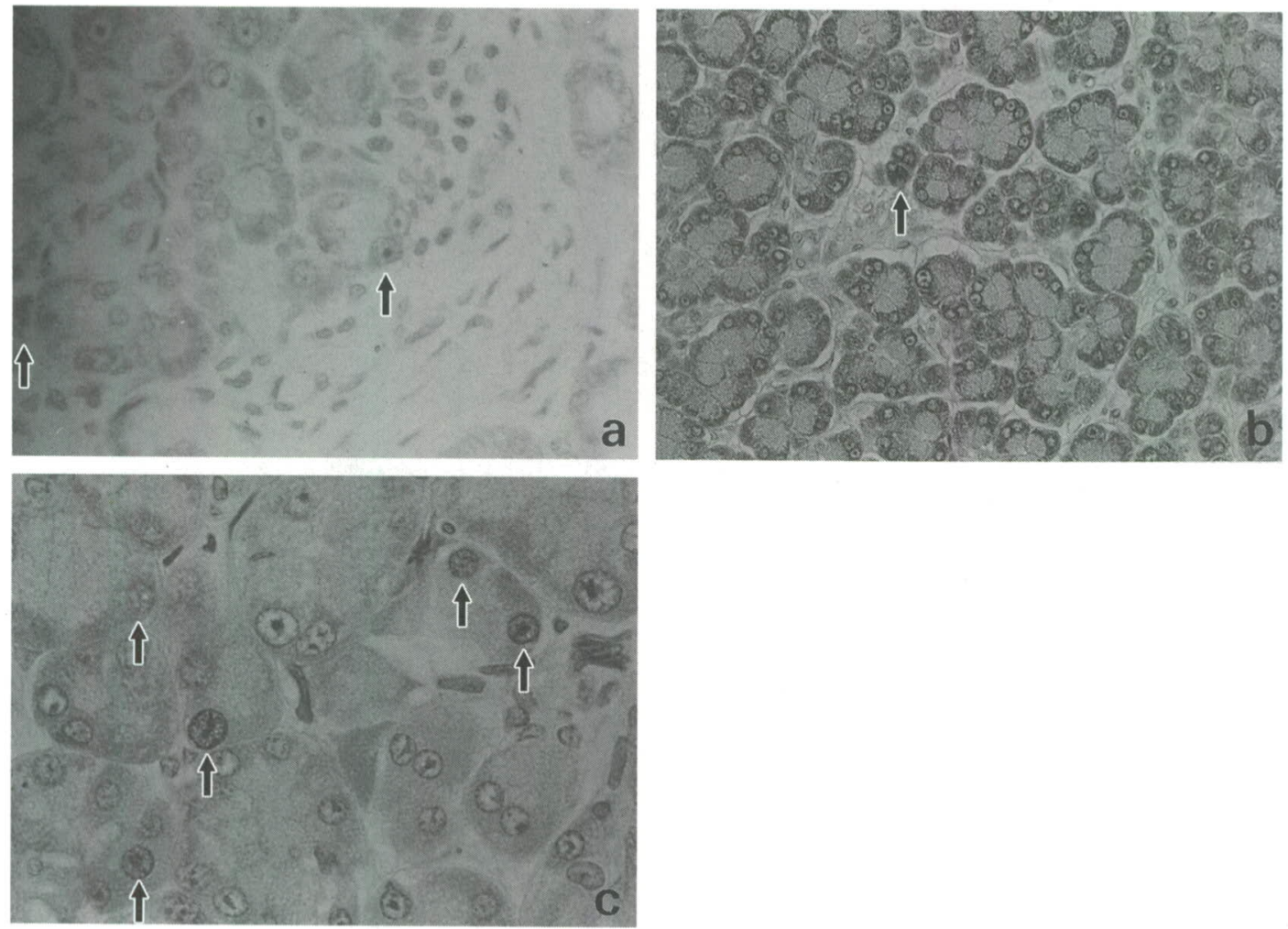

Fig. 4 Histochemical examination of the uptake of Brd-Uridine $(15 \mathrm{mg} / \mathrm{kg})$ in acinar cells of pancreas in the progression stage in the male WBN/Kob rats. $\uparrow$ indicate the cell in which BrdU was uptaken. (a) shows an acinar cell adjacent to the inflammatory area of the nontreated pancreas, which Brd-U is uptaken $(\times 400)$. (b) shows few uptake of Brd- $U$ in the intact acinar cells after 8 weeks of administration of camostat mesylate $(100 \mathrm{mg} / \mathrm{kg} / \mathrm{day})$ from 18 to 25 weeks $(\times 200)$. (c) shows acinar cells after 7 days of administration of camostat mesylate in which Brd-U uptake was increased $(\times 400)$.

acinar cells was not observed in the allopurinoltreated group (Fig. $3-$ e), resulting in no significant increase in the weight of the pancreas compared to that of the non-treated group (Table 3). No significant differences in the levels of PFD, pancreatic enzyme series or IRI were observed between the treated and nontreated groups (Table 3 ).

\section{3 ) Study of pancreatic regeneration}

(1) Expression and distribution of cells exhibiting BrdU uptake : The distribution of cells exhibiting BrdU uptake was noted in isolated and dispersed acinar cells which was in the area of extensive inflammation and fibrosis obser- ved in the pancreas of the nontreated $\mathrm{WBN}$ rats at 18 weeks old (Fig. 4-a). These observations indicated the regeneration and proliferation of acinar cells in the damaged region. On the other hand, after peroral administration of camostat mesylate, which is a trypsin inhibitor, for 8 weeks in rats from 18 weeks old, BrdU uptake into regenerated and proliferated acinar cells was not observed (Fig. 4-b).

(2) Time course of pancreatic regeneration after administration of drugs: To determine the time and area at which the pancreas regenerated following the peroral administration of the trypsin inhibitor, morphological 
changes in the pancreas after camostat mesylate administration $(100 \mathrm{mg} / \mathrm{kg}$ body weight/day) with time were observed. The weight of the pancreas at $0,3,7,14,21,28$ and 42 days after the administration was $802.2 \pm$ $42.6 \mathrm{mg}, \quad 918 \pm 74.8 \mathrm{mg}, \quad 1284.1 \pm 397.5 \mathrm{mg}$, $1160.8 \pm 32.4 \mathrm{mg}, \quad 1055.4 \pm 50.5 \mathrm{mg}, \quad 1407.2 \pm$ $186.4 \mathrm{mg}$ and $1422.9 \pm 154.6 \mathrm{mg}$, respectively. The weight rapidly increased from the 7 th day after the administration, and then gradually increased from the 14th day until the 42nd week with fluctuations. Histopathologically, BrdU uptake in cells was observed in many specimens 7 days after the administration (Fig. 4-c), indicating that a rapid pancreatic proliferation occurred approximately 7 days after camostat mesylate administration. The area in which BrdU uptake was most notably observed was composed of acinar cells adjacent to the borderline between damaged and undamaged areas.

\section{Discussion}

Caerulein-induced pancreatitis, cholinedeficiency pancreatitis and pancreatitis with a ligated pancreatic duct are representative acute pancreatitis models generated experimentally. These models characterized by acute inflammatory changes such as edema, hemorrhage and inflammatory cellular infiltration exhibit severe and fatal extent of pancreatic damage. Pancreatitis induced by long-term administration of $20 \%$ ethanol, ethionine-induced pancreatitis and pancreatitis involving incomplete ligation of the pancreatic duct with a microsphere have been employed as chronic pancreatitis models ${ }^{7,8)}$, all of which merely represent the initial stages of human chronic pancreatitis. Under such circumstances, we ${ }^{2}$ reported the use of the male WBN rat as a spontaneously occurring chronic pancreatitis model. A series of our pathological findings satisfied the histopathologically determined criteria proposed at the Marseille Conference ${ }^{3)}$ (1984) and the histopathologically determined clinical diagnostic criteria of chronic pancreatitis proposed by the Japanese Society of Gastroenterology ${ }^{4}$ (1983). Therefore, our series of rats were determined to exhibit the same pathological changes as those in human chronic pancreatitis. Other than the pathological observations which satisfied the diagnostic criteria proposed by the Japanese Society of Gastroenterology (1983), our findings which were very similar to those on human pancreatic ducts were observed by staining the pancreatic duct with a contrast medium. These changes were very frequently observed (almost 99\%) in male WBN rats from 3 months old, and the severity of the disease and the pattern of progress were almost identical. Accordingly, clarification of mechanisms underlying the development of pancreatitis in WBN rats will profoundly contribute to the clarification of the development and progression of human chronic pancreatitis, and the development of treatment for human pancreatitis, which remain unclarified at present.

Several drugs were administered to WBN rats to treat lesions that are similar to chronic pancreatitis during the initial stage of pancreatitis. Estradiol, camostat mesylate and allopurinol inhibited the development of pancreatitis. Nakama et $\mathrm{al}^{9}$ have already reported that twice-a-week long-term administration (subcutaneous) of $40 \mu \mathrm{g} /$ body estradiol in rats from 2 to 12 months old or the removal of testicles inhibits the development of diabetic mellitus and progression of pancreatic lesions. Similarly, in the present study, administration of the same amount of estradiol as used in our colleagues' study or a smaller amount during the initial stage of pancreatitis could inhibit the 
development of pancreatic lesions. Given the finding that pancreatic lesions progressed in male WBN rats but not in female WBN rats, and the onset of the initial stage of pancreatic lesion corresponded to the sexual maturation stage, it is reasonable to assume that a female hormone at this stage is effective in inhibiting the development of pancreatic lesions. By removing the testicles, pancreatitis was alleviated ${ }^{9}$, however LH-RH was not effective in inhibiting the development of pancreatic lesions. Since the testosterone level in blood decreased to a level similar to that in rats without testicles following LH-RH administration, this female hormone plays the main role in inhibiting the development and progression of pancreatitis. It remains unresolved when and by what mechanisms this female hormone acts on the pancreas of WBN rats.

Peroral administration of camostat mesylate, which is effective in treating chronic pancreatitis as a trypsin inhibitor during the initial stage of pancreatitis, inhibited the development of pancreatitis in WBN rats and resulted in significant improvements of the pancreatic exocrine function. This effect is considered to be attributed to the histologically observed hypertrophy of acinar cells and the resultant increase in the weight of the pancreas. A perorally administered trypsin inhibitor, such as camostat mesylate, is known to have an anti-trypsin effect and a trophic effect ${ }^{10)}$ on pancreatic acinar cells through CCK, based on the observed blockade of the negative feedback mechanism. $\mathrm{We}^{11)}$ had already reported that peritoneal administration of urinastatin which only has an anti-trypsin effect, in rats from 8 to 11 weeks old (the initial period of the development of pancreatic lesions) could inhibit the development of pancreatitis, indicating that trypsin is the prime cause of the development of pancre- atic lesions such as edema, hemorrhage and inflammatory cellular infiltration in WBN rats.

Recently, involvement of activated oxygen in inflammation has been suspected. There is a report describing that a drug that removes activated oxygen is effective at preventing the development of experimentally induced acute pancreatitis ${ }^{12)}$. In this study, to remove activated oxygen, SOD at a dose of $50 \mathrm{mg} / \mathrm{kg}$ body weight and catalase at a dose of $10 \mathrm{mg} / \mathrm{kg}$ body weight were subcutaneously administered twice a week, but they were unable to prevent the development of pancreatitis in WBN rats. Before combining SOD and catalase, the concentrations at which they were administered were determined based on a previous report ${ }^{12)}$ on the inhibition of caerulein-induced pancreatitis. However, since the effective time of SOD inside the body was short, the administration of SOD twice a week could not sufficiently remove activated oxygen. In addition, there were initially low amounts of white-cell-derived $\mathrm{NADPH}$ oxidase, which is an activated oxygen source; this may be the cause of the ineffectiveness of SOD. Meanwhile, allopurinol which is a hypoxanthine-xanthine oxidase inhibitor, exhibited an anti-inhibition effect against the development of pancreatitis, indicating that hypoxanthine-xanthine oxidase system-derived activated oxygen in the pancreatic tissue is involved in the development of pancreatitis.

The next step in this study is to select and evaluate drugs which are safe and clinically applicable for treating pancreatitis at the progression stage from among the drugs which inhibited the development of WBN pancreatic lesions in the screening test. A summary of results for the treatment administration is as follows. Peroral administration of camostat mesylate was significantly effective at treating 
pancreatitis both in the initial and progression stages; peroral administration of allopurinol was significantly effective in the initial stage, while it was not effective in the progression stage. Results of this study suggest that both trypsin and activated oxygen are involved in the initial stage of pancreatitis in male WBN rats. Meanwhile, only peroral administration of a trypsin inhibitor was effective against chronic lesions, mainly pancreatic fibrosis and atrophy which occurred during the progression stage. This suggests that rather than the suppression of the activated oxygen and anti-trypsin effects, the pancreatic trophic effect, which is based on pancreozymin secretion activation due to blockage of the negative feedback mechanisms by peroral administration of a trypsin inhibitor, is required for treatment of chronic pancreatitis during the progression stage. To substantiate this suggestion, we studied the expression of cells at the DNA synthesis stage based on the uptake of BrdU. We found that the uptake of BrdU was notable in the acinar cells around the borderline between damaged and undamaged areas, which was not observed after the pancreas was regenerated 2 months after camostat mesylate administration. Accordingly, we determined that when an undamaged acinus which remained in the severely chronic pancreatitic lesions expanded following regeneration of the pancreas, the mechanism through which a drug is effective against chronic pancreatitis could be observed. In other words, it is appropriate to consider that a biophylaxis against destruction of exocrine cells exists in damaged areas. To this system, regeneration of the pancreas via pancreozymine is achieved by peroral administration of a trypsin inhibitor. Since a rapid increase in the weight of the pancreas from the 7th day after administration of camostat mesylate occurred concurrently with the growth of cells exhibiting BrdU uptake in specimens 7 days after administration, pancreatic regeneration was mostly improved about 1 week after administration of camostat mesylate. At present, camostat mesylate is the only drug which is commercially available for treating chronic pancreatitis. In this study, the treatment efficacy of camostat mesylate was confirmed in the WBN rat, which strongly supports the appropriateness of this model and its similarity to humans.

Since there is as yet no appropriate chronic pancreatitis model, determination of the efficacy of drugs for pancreatitis treatment has been performed using artificially developed pancreatitis models such as pancreatitis with ligated pancreatic ducts and ethionine-induced pancreatitis. Using these models, the survival rate was mainly determined. This study could determine whether or not pancreatitis is indeed treated by test drugs using spontaneously occurring male WBN rats. As suggested by the results of allopurinol administration in this study, the efficacy of drugs varied depending on the stage of the disease at which the drugs were administered. If human chronic pancreatitis develops through two stages consisting of the initial stage with acute inflammation and the progression stage with fibrosis, drugs applicable for treating the disease when it is in the initial stage and those for treating the disease in the chronic stage are considered to be different. Evaluation of drugs for chronic pancreatitic treatment is considered to necessitate the use of 3 groups of rats : group I to which drugs are administered during the initial stage, group II to which drugs are administered during the progression stage and group III to which drugs are withheld after administration during the initial stage; the clinical efficacy of drugs in each group should be evaluated. Group III 
was needed in our studies ${ }^{13,14)}$ because the determination of whether or not a drug for chronic pancreatitis administeredduring the initial stage inhibits subsequent progression of pancreatitis is clinically significant.

By what index the efficacy of drugs is evaluated is an important issue. The serum level of pancreatic enzyme and the PFD level clinically reflect the pancreatic exocrine ability to some extent, however, since these measurements are unreliable in an animal study, it may poorly reflect the efficacy of a drug. As clarified in this study, the most appropriate index for evaluating the efficacy of a drug for chronic pancreatitis in WBN rats is based on histopathological observation of the pancreas. The percentage of pancreatitic area is effective for comparing observations of pathological findings quantitatively.

Pancreatic biopsy in the actual clinical field is almost impossible, therefore how chronic pancreatitis progresses and the effectivity of the drugs with time cannot be studied. Under such circumstances, it is concluded that the WBN rat is a good chronic pancreatitis model that is highly effective not only for studying the progression of the disease from chronic pancreatitis, but also for pharmacological studies of drugs for chronic pancreatitis.

\section{Conclusion}

(1) A new evaluation method for the treatment of chronic pancreatitis using the WBN/ Kob rat as a human chronic pancreatitis model was determined.

(2) As a result of screening for drugs that inhibit the development of pancreatic lesions in WBN rats, the inhibitory effects of estradiol, camostat mesylate and allopurinol were observed.

(3) The efficacy of camostat mesylate and allopurinol which might be clinically used for progressive pancreatitis was studied. Camostat mesylate was found to be effective, but not allopurinol.

(4) The expression of cells at the DNA synthesis stage as determined based on BrdU uptake was examined. The uptake of BrdU notably occurred in acinar cells adjacent to the pancreatitic area, while few cells exhibiting BrdU uptake were observed in the regenerated pancreatic tissue after administration of camostat mesylate for 2 months.

(5) The regeneration of the pancreas as a result of the pancreatic trophic effect is the mechanism through which camostat mesylate is effective against lesions (mainly fibrosis) of chronic pancreatitis during the progression stage. One week after camostat mesylate administration, the weight of the pancreas increased rapidly, during which the uptake of BrdU in cells increased concurrently, indicating that regeneration of the pancreas occurred during this period.

(6) The most effective parameter for evaluating the efficacy of a drug for pancreatitis is the percentage of pancreatitic area employed in our histopathological study. It was possible to evaluate drugs for chronic pancreatitis treatment using this parameter.

Accordingly, we concluded that the use of $\mathrm{WBN} / \mathrm{Kob}$ rats is effective for drug evaluation in the treatment of chronic pancreatitis.

\section{References}

1) Nakama, K., Shichinohe, K., Naito, K., et al. : Spontaneous diabetes-like syndrome in WBN/ Kob rats. Acta Diabetol. Lat., 22 : 335-342 (1985).

2) Ohashi, K., Kim, J. H., Hara, H., et al. : WBN/ $\mathrm{KoB}$ rats. Spontaneously occuring model of chronic pancreatitis. Int. J. Pancreatol., $6: 231-$ 247 (1990).

3) Gyr, K. E., Siger, M. V., Sarles, H., eds. : Revised 
classification of pancreatitis-Marseille, 1984. : The participants of the Second International Symposium on the Classification of Pancreatitis agreed on a revised classification of pancreatitis, "Pancreatitis-Concepts and Classification", Elsevier, Amsterdam, 1984.

4) "Clinical diagnostic criteria of chronic pancreatitis" Proposed by the Japanese. Society of Gastroenterology. the Jpn. J. Gastroenterology, $80: 1863-1866$ (1983) (in Japanese).

5) Yamato, C. and Kinoshita, K. : A simple assay for measurement of urinary $\mathrm{p}$-aminobenzoic acid in the oral pancreatic function test. Analyt. Biochem., 98 : 13-17 (1979).

6) Sasaki, k., Ogino, T. and Takahashi, M.: Immunological determination of labeling index on human tumor tissue section using monoclonal anti-Brd. Urd antibody. Stain. Technol., 61 : 155161 (1986).

7) Steer, L. M.: Workshop on experimental pancreatitis. Dig. Dis. Sci. 30 : 575-581 (1985).

8) Kinami, Y. and Kita, I. : Experimental model of chronic pancreatitis. Igaku no Ayumi, 144: 421423 (1988) (in Japanese).

9) Nakama, K., Akimoto, T., Shimizu, M., et al. : A preventional effect of estrogen on the development of spontaneously occuring diabetes mellitus in male WBN/Kob rat. Diabetic animal $2:$ ed by Yoshio Gotoh, IYAKU Journal, Osaka, 130-136, 1988 (in Japanese)

10) Green, G. M. and Lyman, R. L. : Feedback regulation of pancreatic enzyme secretion as a mechanism for trypsin inhibitor-induced hypersecretion in rats. Proc. Soc. Exp. Biol. Med. $140: 6-12,1972$

11) Nakama, K., Akimoto, T., Kim, J. H., et al. : Effect of urinastantin (MIRACLID) on spontaneous pancreatitis in WBN/Kob rats-A pathomorphological study-. Gallbladder and Pancreas, 11: 547-553 (1990) (in Japanese).

12) Sanfey, H., Bulkley, G. B. and Cameron, J. L. : The role of oxygen-derived free radicals in the pathogenesis of acute pancreatitis. Ann. Surg., 200 : 405-413 (1984).

13) Nakama, K., Akimoto, T. and Ohashi, K.: Histopathological study of the effect of FUT-187 on the spontaneous chronic pancreatitis in WBN/ Kob Rats. Gallbladder and Pancreas, 16 : 443450 (1995) (in Japanese).

14) Ohashi, K., Hara, H., Aso, R., et al. : Pancreatic enzyme study on the anti-pancreatic effect of FUT-187 on spontaneous chronic pancreatitis in WBN/Kob rats. Gallbladder and Pancreas, 16 : 531-536 (1995) (in Japanese). 